\title{
Predicting meningioma consistency and brain-meningioma interface with intraoperative strain ultrasound elastography: a novel application to guide surgical strategy
}

\author{
*Giuseppe Maria Della Pepa, MD,1 Grazia Menna, ${ }^{1}$ Vito Stifano, MD,, Angelo Maria Pezzullo, MD, ${ }^{2}$ \\ Anna Maria Auricchio, MD, ${ }^{1}$ Alessandro Rapisarda, MD, ${ }^{1}$ Valerio Maria Caccavella, ${ }^{1}$ \\ Giuseppe La Rocca, MD, ${ }^{1}$ Giovanni Sabatino, MD, PhD, ${ }^{1}$ Enrico Marchese, MD, ${ }^{1}$ and \\ Alessandro Olivi, MD'
}

\begin{abstract}
${ }^{1}$ Institute of Neurosurgery and ${ }^{2}$ Public Health Department, Fondazione Policlinico Universitario Agostino Gemelli IRCCS, Catholic University, Rome, Italy
\end{abstract}

\begin{abstract}
OBJECTIVE Providing new tools to improve surgical planning is considered a main goal in meningioma treatment. In this context, two factors are crucial in determining operating strategy: meningioma-brain interface and meningioma consistency. The use of intraoperative ultrasound (ioUS) elastosonography, a real-time imaging technique, has been introduced in general surgery to evaluate similar features in other pathological settings such as thyroid and prostate cancer. The aim of the present study was to evaluate ioUS elastosonography in the intraoperative prediction of key intracranial meningioma features and to evaluate its application in guiding surgical strategy.
\end{abstract}

METHODS An institutional series of 36 meningiomas studied with ioUS elastosonography is reported. Elastographic data, intraoperative surgical findings, and corresponding preoperative MRI features were classified, applying a score from 0 to 2 to both meningioma consistency and meningioma-brain interface. Statistical analysis was performed to determine the degree of agreement between meningioma elastosonographic features and surgical findings, and whether intraoperative elastosonography was a better predictor than preoperative MRI in assessing meningioma consistency and slip-brain interface, using intraoperative findings as the gold standard.

RESULTS A significantly high degree of reliability and agreement between ioUS elastographic scores and surgical finding scores was reported (intraclass correlation coefficient $=0.848, F=12.147, p<0.001$ ). When analyzing both consistency and brain-tumor interface, ioUS elastography proved to have a rather elevated sensitivity, specificity, positive predictive value (PPV), negative predictive value (NPV), and positive (LR+) and negative likelihood ratio (LR-). This consideration was true especially for meningiomas with a hard consistency (sensitivity $=0.92$, specificity $=0.96$, PPV = $0.92, \mathrm{NPV}=0.96, \mathrm{LR}+=22.00, \mathrm{LR}-=0.09$ ) and for those presenting with an adherent slip-brain interface (sensitivity = 0.76 , specificity $=0.95, \mathrm{PPV}=0.93, \mathrm{NPV}=0.82, \mathrm{LR}+=14.3, \mathrm{LR}-=0.25)$. Furthermore, predictions derived from ioUS elastography were found to be more accurate than MRI-derived predictions, as demonstrated by McNemar's test results in both consistency $(p<0.001)$ and interface $(p<0.001)$.

CONCLUSIONS While external validation of the data is needed to transform ioUS elastography into a fully deployable clinical tool, this experience confirmed that it may be integrated into meningioma surgical planning, especially because of its rapidity and cost-effectiveness.

https://thejns.org/doi/abs/10.3171/2020.10.FOCUS20797

KEYWORDS elastography; meningioma; intraoperative ultrasound; strain ultrasound elastography

ABBREVIATIONS $\mathrm{Cl}=$ confidence interval; ICC = intraclass correlation coefficient; ioUS = intraoperative US; LR+ = positive likelihood ratio; LR- = negative likelihood ratio; MRE = MR elastography; NPV = negative predictive value; PPV = positive predictive value; RF = radiofrequency; SUE = strain ultrasound elastography; US = ultrasound. SUBMITTED September 1, 2020. ACCEPTED October 22, 2020.

INCLUDE WHEN CITING DOI: 10.3171/2020.10.FOCUS20797.

* G.M.D.P. and G.M. contributed equally to this work. 
$\mathrm{M}$ ENINGIOMAS are brain tumors arising from the arachnoid cells and represent the most common primary intracranial neoplasm. Despite the survival benefit associated with novel therapeutic approaches, surgery currently remains the mainstay of treatment. In this context, two factors are crucial in determining surgical strategy: meningioma-brain interface and meningioma consistency. ${ }^{1}$

Only a few imaging methods are used to predict these two determinants, mainly in a preoperative setting. CT and MRI are routinely used, but may only provide an approximate assessment of the dynamic relationship between the meningioma and its surroundings. ${ }^{2}$ Despite the feasibility and utility of the aforementioned imaging methods, a realtime imaging technique that could intraoperatively identify the consistency of intracranial pathology would be of paramount importance to assess a safe plane of dissection and to provide a real-time guide in meningioma resection. In this context, elastosonography opens up innovative possibilities.

Pioneering studies in the literature proved the integration of ultrasound (US) and elastosonography to be valuable: intraoperative US (ioUS) elastosonography has been developed to predict tissue stiffness and successfully evaluates tissue mobility and extracapsular invasion in thyroid nodules and in prostate cancer, ${ }^{3}$ with potential applications to neurosurgery as well. ${ }^{4}$

The study presented in this paper is the first attempt to translate these experiences into another context, namely meningioma surgery. An institutional series of 36 meningiomas studied with ioUS elastosonography is reported. The aim of the present study is to assess whether elastosonographic characteristics are predictive of key intracranial meningioma features, and how those meningioma features can be used as real-time guidance to improve surgical strategy. Limitations and possible future implications of its implementation in a large-scale setting will be considered in detail and discussed as well.

\section{Methods \\ Study Population}

We prospectively enrolled 36 patients who underwent surgery for a histopathologically confirmed diagnosis of intracranial meningioma between March 2018 and July 2020. The IRB of Catholic University approved the use of intraoperative elastography as part of the standard US examination. Therefore, this study was based on anonymized data with a waiver of informed consent. All procedures were performed in accordance with the ethical standards of the institutional and/or national research committee and with the 1964 Helsinki declaration and its later amendments or comparable ethical standards.

Intraoperative US elastographic maps were acquired after craniotomy and before dural opening, exploiting spontaneous brain pulsation. The following relevant patient characteristics were collected: 1) demographic features, such as age at diagnosis (in years) and sex; 2) tumor characteristics, i.e, location, side, size (cm), and edema; and 3) histopathological features, such as variants, presence of calcifications, number of mitoses, and presence of necrosis.

\section{Study Design}

Intraoperative Assessment

After the craniotomy and before the dural opening, the surgical field was examined with US (MyLabTwice, a linear-array multifrequency $3-11 \mathrm{MHz}$ device) through the craniotomy window, using a probe covered by a sterile plastic sheet with sterile US coupling gel. B-mode was first used to correctly identify proper anatomy. An elastography map was then obtained using the strain ultrasound elastography (SUE) modality and automatically superimposed to the B-mode images. Neurosurgeon findings during the resection were considered the gold standard. All dissection features, including meningioma consistency and brain-tumor interface, were recorded.

\section{Postoperative Analysis}

The obtained results were later matched to the corresponding preoperative MRI features; the latter were retrospectively analyzed by experienced investigators, who were blinded to the intraoperative findings. Thus, we statistically assessed: 1) the degree of agreement between meningioma elastosonographic features and surgical findings with regard to meningioma consistency and meningioma-brain interface; and 2) whether intraoperative elastosonography was a better predictor than preoperative MRI in assessing meningioma consistency and slip-brain interface, using intraoperative findings as the gold standard.

\section{Strain Ultrasound Elastography}

In our setting, we exploited spontaneous brain pulsations to produce the elastographic map after craniotomy and before dural opening. The rationale is that the SUE map is predictive of the consistency of the meningioma (depicting the overall meningioma texture as well as softer/harder areas within the tumor), and of the features of the meningioma-brain interface (the presence of stiffer peritumoral areas due to extracapsular spreading and tumor adherences, as well as a relative "softness" due to the presence of CSF film). ${ }^{5}$

A US radiofrequency (RF) waveform is a plot of voltage produced following excitation of a piezoelectric material within a US probe by returning US waves, against time. Return time for a given wave is dependent on the depth of the material that reflected that wave, assuming the speed of sound is constant throughout the scan plane. Conventional US imaging displays the RF amplitude, which is related to the intensity of the returning wave, against time or, indirectly, depth. Hence, a US image depicts the acoustic reflectivity of a scanned area. ${ }^{6}$

Following spontaneous brain pulsations, the subsequent tissue displacement is tracked between pairs of RF echo frames and the strain calculated from the axial gradient of the displacements. Under an equal amount of stress, a stiff region experiences less strain (deformation) than surrounding softer tissue. Using a color map to code different magnitudes of strain, a 2D strain image can be translucently superimposed on the conventional B-mode image, aiding the assessment of the spatial relationship between the US image and the elastographic data. Quantitative elasticity measurements expressed in $\mathrm{kPa}$ cannot be obtained, 
as the local degree of stress is unknown. Thus, strain elastography is a qualitative technique in which relative stiffness differences in insonated tissue are displayed in a color map, in which the reddest coloration represented the softest elasticity, and the bluest represented the stiffest (Fig. 1).

\section{Data Extraction}

Intraoperatively obtained elastographic data were collected from each patient and retrospectively analyzed by experienced investigators. Because an elastosonographic map is not quantitative, to ease data collection, elastographic features were categorized using a score from 0 to 2, as follows: 1) for meningioma consistency, $0=\mathrm{soft}$ consistency, 1 = intermediate consistency, and $2=$ hard consistency; and 2) for meningioma-brain interface (or slip-brain interface), $0=$ preserved meningioma-brain interface, 1 = mixed meningioma-brain interface, and $2=$ adherent meningioma-brain interface. Similarly, a score from 0 to 2 was assigned to the corresponding preoperative MRI features (T2-weighted sequences) and to the intraoperative surgical findings.

\section{Operative Setup}

All surgeries were performed with an OPMI Pentero (Carl Zeiss) or a Leica M720 OH5 (Leica Microsystems) microscope. Electrophysiological monitoring (Nicolet Endeavor CR, Cardinal Health) and a neuronavigation system were used when appropriate. A Cavitron ultrasonic surgical aspirator was used in most procedures, according to the surgeon's request.

\section{Statistical Analysis}

Means and percentages were generated for continuous and discrete variables, where appropriate. The intraclass correlation coefficient (ICC) two-way fixed-effects ANOVA model for absolute agreement (ICC[3,1]) was computed to evaluate and quantify the degree of reliability of both US elastography and MRI scores in predicting intraoperatively assessed meningioma consistency and brain-tumor interface. The ability of both ioUS and preoperative T2-weighted imaging to correctly predict meningioma consistency and interface before surgery was evaluated by computing the following performance metrics: sensitivity, specificity, positive and negative predictive values (PPV and NPV), positive likelihood ratio ( $\mathrm{LR}+$ ), and negative likelihood ratio (LR-), with $95 \%$ confidence intervals (CIs) being calculated for each score. McNemar's test was used to further investigate differences in the overall discriminative ability and predictive validity of both imaging tools. Statistical significance was considered at a 2 -tailed $\mathrm{p}$ value $<0.05$. All analyses were performed using $\mathrm{R}$ (version 3.6.3, The R Foundation for Statistical Computing).

\section{Results}

In the analyzed period, 36 patients ( 21 females [58.3\%], 15 males [41.7\%], mean age 61.2 years, age range $23-79$ years) were included for the present study. Clinical characteristics are summarized in Table 1.

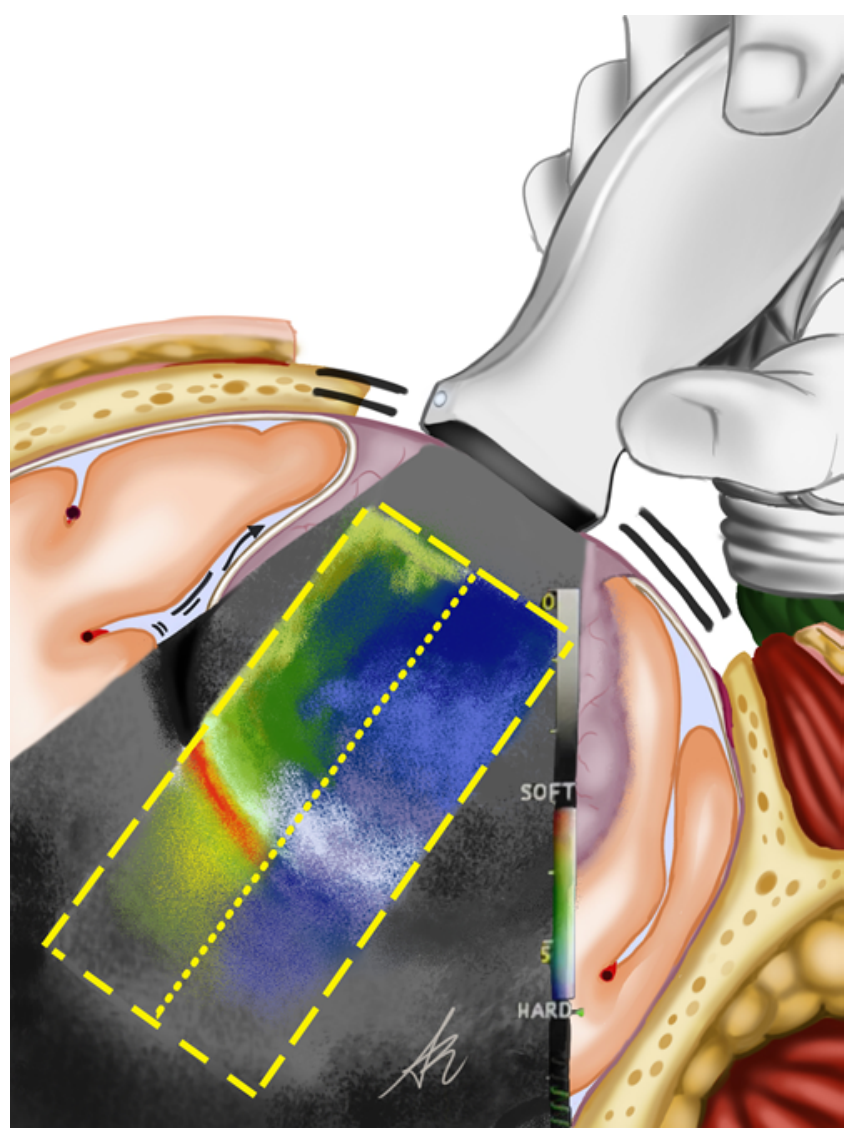

FIG. 1. Illustration summarizing the rationale behind our work. Elastography is a sonographically based real-time strain imaging method for registering the elastic properties of tissues, with promising application in meningioma surgery. In our setting, spontaneous brain pulsations are used to generate the elastographic map after craniotomy and before dural opening. The difference in strain is determined by measuring the local deformation of the tissue when it is compressed. We aimed to evaluate whether elastography could be used to real-time evaluate consistency and brain-tumor interface via a color-coded map. In this case, the maps represent a qualitative visualization of the relative "elasticity" of tissue. This means deformation of harder tissue is lower than that of softer tissue: the first is represented by dark colors (blue), whereas "softer" tissues are displayed in red. This consideration holds true also for the interface: if there is CSF within a subarachnoid space outside the tumor and it is free to move, the interface is displayed in red; if there is a loss of arachnoid film due to tumor infiltration, the brain-tumor interface appears blue. The drawing is split into two parts: in the left side of the dashed rectangle there is a lesion presenting with good interface (red) and soft consistency (green), while in the right side a hard-consistency tumor (blue), characterized by the presence of an adherent interface (blue), is depicted. Elasticity cannot be quantitatively measured with this method; it is only possible to determine a color map depicting relative stiffness differences in insonated tissues. Copyright Alessandro Rapisarda. Published with permission.

An excellent and statistically significant degree of agreement was found between US elastographic predictions and surgical findings in term of both meningioma consistency ( ICC $=0.848, \mathrm{p}<0.001$ ) and brain-tumor interface $(\mathrm{ICC}=0.845, \mathrm{p}<0.001)$. Conversely, poor agreement was reported between preoperative MRI predictions and surgical findings (meningioma consistency $\mathrm{ICC}=$ 
TABLE 1. Characteristics of the 36 patients in the study

\begin{tabular}{|c|c|}
\hline Characteristic & Value \\
\hline \multicolumn{2}{|l|}{ Age, yrs } \\
\hline Mean (SD) & $61.2(13.6)$ \\
\hline Median (min, max) & $65.0(23.0,79.0)$ \\
\hline \multicolumn{2}{|l|}{ Sex, n (\%) } \\
\hline $\mathrm{F}$ & $21(58.3)$ \\
\hline M & $15(41.7)$ \\
\hline \multicolumn{2}{|l|}{ Location, n (\%) } \\
\hline Ethmoidal & $1(2.8)$ \\
\hline Falx tentorium & $4(11.1)$ \\
\hline Frontal & $10(27.8)$ \\
\hline Intraventricular & $2(5.6)$ \\
\hline Occipital & $2(5.6)$ \\
\hline Parietal & $7(19.4)$ \\
\hline Sphenoid wing & $3(8.3)$ \\
\hline Temporal & $5(13.9)$ \\
\hline Tentorium & $2(5.6)$ \\
\hline \multicolumn{2}{|l|}{ Side, $n(\%)$} \\
\hline Bilat & $1(2.8)$ \\
\hline $\mathrm{Lt}$ & $15(41.7)$ \\
\hline Rt & $20(55.6)$ \\
\hline \multicolumn{2}{|l|}{ Tumor size, $\mathrm{cm}$} \\
\hline Mean (SD) & $4.09(1.16)$ \\
\hline Median (min, max) & $4.05(1.80,7.60)$ \\
\hline \multicolumn{2}{|l|}{ Edema, n (\%) } \\
\hline No & $18(50.0)$ \\
\hline Yes & $18(50.0)$ \\
\hline \multicolumn{2}{|c|}{ Histological variants, $\mathrm{n}(\%)$} \\
\hline Anaplastic & $5(13.9)$ \\
\hline Atypical & $11(30.6)$ \\
\hline Meningothelial & $20(55.6)$ \\
\hline \multicolumn{2}{|l|}{ Calcifications, n (\%) } \\
\hline No & $18(50.0)$ \\
\hline Yes & $18(50.0)$ \\
\hline \multicolumn{2}{|l|}{ Mitosis* } \\
\hline Mean (SD) & $5.50(6.29)$ \\
\hline Median (min, max) & $5.00(0,22.0)$ \\
\hline \multicolumn{2}{|l|}{ Necrosis, $n(\%)$} \\
\hline No & $33(91.7)$ \\
\hline Yes & $3(8.3)$ \\
\hline
\end{tabular}

${ }^{*}$ Mitosis in 10 consecutive high-power fields.
$0.006, p=0.486$; brain-tumor interface ICC $=0.418, p=$ $0.006)$, indicating low reliability of this imaging modality (Table 2).

In predicting consistency, ioUS elastography reported high sensitivity in all grades, especially when tumor consistency was hard (score $0=0.86,95 \%$ CI $0.42-1.00$; score $1=0.82,95 \%$ CI $0.57-0.96$; score $2=0.92,95 \%$ CI 0.62-1.00; Table 3). Interestingly, a weak positive but statistically significant correlation $(r=0.53, p<0.001)$ was found between histological grade and meningioma-brain interface assessed via US elastosonography. Regarding consistency, MRI showed a lower sensitivity (score $0=$ $0.71,95 \%$ CI $0.29-0.96$; score $1=0.41,95 \%$ CI $0.18-0.67$; score $2=0.17,95 \%$ CI $0.02-0.48$ ), especially for hardconsistency meningiomas. Moreover, MRI reported low PPVs (score $0=0.26,95 \%$ CI $0.09-0.51$; score $1=0.58$, 95\% CI 0.28-0.85; score $2=0.40,95 \%$ CI $0.05-0.85$ ). LR+ values should be noted as well; for US elastography they were 12.43 (95\% CI 3.15-48.96), 7.82 (95\% CI 2.07-29.56), and 22.00 (95\% CI 3.21-151.00) for score 0, 1 , and 2, respectively. MRI showed the following matched results: 1.48 (95\% CI 0.81-2.70), 1.56 (95\% CI 0.61-4.02), and 1.33 (95\% CI 0.26-6.94). Similar findings can be reported when considering the slip-brain interface predictions, in which US elastography reported high sensitivity and overall good PPV, LR+, and LR- (Table 3).

The overall superiority of ioUS elastography was further confirmed by the results of McNemar's test. The test showed a significant difference in both meningioma consistency $(\mathrm{p}<0.001)$ and brain-tumor interface $(\mathrm{p}<0.001)$ scores produced by ioUS elastography over matched MRI scores when surgical finding scores were the gold standard (Table 4).

\section{Discussion}

Consistency and slip-brain interface are major determinants in defining a meningioma surgical strategy, especially if considered in relation to critical cortical areas and vascular structures. Precise knowledge of these two features may thus guide operative decisions step by step. Consequently, an intraoperative real-time imaging modality capable of identifying these key features before dissection starts may prove to be a valuable guide.

We will first discuss available and approved tools, focusing on MR elastography (MRE) and ioUS elastography in fields other than neurosurgery; against this background, we will analyze the added value of ioUS elastography in meningioma surgery, with a special focus on previous experiences in brain tumor surgery, reliability of SUE maps, and their significance. Lastly, the implications and limita-

TABLE 2. ICC for interrater agreement between ioUS, MRI predictions, and intraoperative surgical findings

\begin{tabular}{|c|c|c|c|c|c|c|}
\hline \multirow[b]{2}{*}{ Interrater Agreement } & \multicolumn{3}{|c|}{ Meningioma Consistency } & \multicolumn{3}{|c|}{ Brain-Tumor Interface } \\
\hline & $\operatorname{ICC}(95 \% \mathrm{Cl})$ & F Value & p Value & $\operatorname{ICC}(95 \% \mathrm{CI})$ & F Value & $p$ Value \\
\hline ioUS vs surgical findings & $0.848(0.731,0.924)$ & 12.147 & $<0.001$ & $0.845(0.715,0.926)$ & 11.937 & $<0.001$ \\
\hline MRI vs surgical findings & $0.006(-0.334,0.347)$ & 1.012 & 0.486 & $0.418(0.103,0.664)$ & 2.437 & 0.006 \\
\hline ioUS vs MRI & $0.101(-0.242,0.423)$ & 1.225 & 0.282 & $0.402(0.083,0.655)$ & 2.344 & 0.008 \\
\hline
\end{tabular}


TABLE 3. Summary of ioUS elastography and MRI versus surgical findings in identifying meningioma consistency and brain-tumor interface before surgery

\begin{tabular}{|c|c|c|c|c|c|c|}
\hline Variable & Sensitivity & Specificity & PPV & NPV & LR+ & LR- \\
\hline \multicolumn{7}{|c|}{ Meningioma consistency } \\
\hline \multicolumn{7}{|c|}{ ioUS elastography score } \\
\hline 0 & $0.86(0.42,1.00)$ & $0.93(0.77,0.99)$ & $0.75(0.35,0.97)$ & $0.96(0.82,1.00)$ & $12.43(3.15,48.96)$ & $0.15(0.02,0.94)$ \\
\hline 1 & $0.82(0.57,0.96)$ & $0.89(0.67,0.99)$ & $0.88(0.62,0.98)$ & $0.85(0.62,0.97)$ & $7.82(2.07,29.56)$ & $0.20(0.07,0.56)$ \\
\hline 2 & $0.92(0.62,1.00)$ & $0.96(0.79,1.00)$ & $0.92(0.62,1.00)$ & $0.96(0.79,1.00)$ & $22.00(3.21,151.00)$ & $0.09(0.01,0.57)$ \\
\hline \multicolumn{7}{|l|}{ MRI score } \\
\hline 0 & $0.71(0.29,0.96)$ & $0.52(0.33,0.71)$ & $0.26(0.09,0.51)$ & $0.88(0.64,0.99)$ & $1.48(0.81,2.70)$ & $0.55(0.16,1.88)$ \\
\hline 1 & $0.41(0.18,0.67)$ & $0.74(0.49,0.91)$ & $0.58(0.28,0.85)$ & $0.58(0.37,0.78)$ & $1.56(0.61,4.02)$ & $0.80(0.49,1.29)$ \\
\hline 2 & $0.17(0.02,0.48)$ & $0.88(0.68,0.97)$ & $0.40(0.05,0.85)$ & $0.68(0.49,0.83)$ & $1.33(0.26,6.94)$ & $0.95(0.71,1.28)$ \\
\hline \multicolumn{7}{|c|}{ Brain-tumor interface } \\
\hline \multicolumn{7}{|c|}{ US elastography score } \\
\hline 0 & $1.00(0.59,1.00)$ & $0.90(0.73,0.98)$ & $0.70(0.35,0.93)$ & $1.00(0.87,1.00)$ & $9.67(3.31,28.22)$ & $0.00(0.00,0.00)$ \\
\hline 1 & $0.67(0.35,0.90)$ & $0.83(0.63,0.95)$ & $0.67(0.35,0.90)$ & $0.83(0.63,0.95)$ & $4.00(1.50,10.66)$ & $0.40(0.18,0.91)$ \\
\hline 2 & $0.76(0.50,0.93)$ & $0.95(0.74,1.00)$ & $0.93(0.66,1.00)$ & $0.82(0.60,0.95)$ & $14.53(2.12,99.68)$ & $0.25(0.10,0.59)$ \\
\hline \multicolumn{7}{|l|}{ MRI score } \\
\hline 0 & $0.43(0.10,0.82)$ & $0.93(0.77,0.99)$ & $0.60(0.15,0.95)$ & $0.87(0.70,0.96)$ & $6.21(1.27,30.40)$ & $0.61(0.32,1.17)$ \\
\hline 1 & $0.41(0.18,0.67)$ & $0.74(0.49,0.91)$ & $0.58(0.28,0.85)$ & $0.58(0.37,0.78)$ & $1.56(0.61,4.02)$ & $0.80(0.49,1.29)$ \\
\hline 2 & $0.18(0.04,0.43)$ & $0.89(0.67,0.99)$ & $0.60(0.15,0.95)$ & $0.55(0.36,0.73)$ & $1.68(0.32,8.86)$ & $0.92(0.70,1.20)$ \\
\hline
\end{tabular}

Data given as value $(95 \% \mathrm{Cl})$.

tions related to a surgical application of our technique will be highlighted.

\section{MRE and ioUS Elastography Applications in Fields Other Than Neurosurgery}

Preoperative assessment of consistency and interface was first attempted with specific MRI, namely MRE, that was developed over the last decade to meet this clinical need. This technique measures the propagation of vibration-induced displacement and the viscoelastic mechanical properties in the tissue of interest. However, the existing literature on MRE is sparse; its application is currently anecdotal and limited to experimental settings. Moreover, the diagnostic accuracy of MRE is further limited by suboptimal imaging, due to the artifact introduced by signal interference of the surrounding bony cranial anatomy. ${ }^{2,3,7,8}$

Within this context, ioUS elastography may play a role as an innovative intraoperative imaging technique. The series presented in this paper borrows a technique previously validated in other oncological pathologies. There are consistent results in the literature concerning SUE in prostate cancer, where "stiffness" in the peritumoral area correlates with extracapsular tumor spreading and tumor adherence to surrounding tissue; conversely, a relative "softness" in the pericapsular areas is indicative of a tumor still confined to the capsule. ${ }^{9}$ Similarly, this principle has been also applied to differentiate extracapsular invasion in breast invasive lobular carcinoma and malignant thyroid nodules. ${ }^{10,11}$ Strong signals indicating soft edges are also frequently seen in abdominal imaging of the pancreas and bowel, representing natural slip surfaces such as the peritoneum or luminal surface of the bowel. ${ }^{12,13}$

\section{ioUS Elastography in Meningioma Surgery}

Recently, some studies have highlighted the potential application of ioUS elastography in brain tumor surgery. This tool may aid the surgeon in quantitatively distinguishing benign from malignant tumors (especially in gliomas), obtaining consistent data by means of consistent pathology..$^{14-17}$ In 2009, Uff et al. ${ }^{18}$ presented for the first time elastosonographic acquisition obtained through arterial pulsations during spinal cord surgery. Their study concluded that SUE data correlate with the surgeon's finding of tissue stiffness. Moreover, areas of lower stiffness at tumor boundaries were linked to the presence of cleavage planes.

TABLE 4. Results of the McNemar test

\begin{tabular}{cccc}
\hline & \multicolumn{2}{c}{ MRI Score } & \\
\cline { 2 - 3 } Category & $\begin{array}{c}\text { Correctly } \\
\text { Identified }\end{array}$ & $\begin{array}{c}\text { Not Correctly } \\
\text { Identified }\end{array}$ & $\begin{array}{c}\text { McNemar's } \chi^{2} \\
(p \text { value })\end{array}$ \\
\hline Meningioma consistency & & & $13.76(<0.001)$ \\
\hline US elastography score & & & \\
\hline Correctly identified & 12 & 19 & \\
\hline $\begin{array}{c}\text { Not correctly identi- } \\
\text { fied }\end{array}$ & 2 & 3 & \\
\hline Brain-tumor interface & & & \\
\hline $\begin{array}{c}\text { US elastography score } \\
\text { Correctly identified }\end{array}$ & 8 & 20 & \\
\hline $\begin{array}{l}\text { Not correctly identi- } \\
\text { fied }\end{array}$ & 2 & 6 & \\
\hline
\end{tabular}



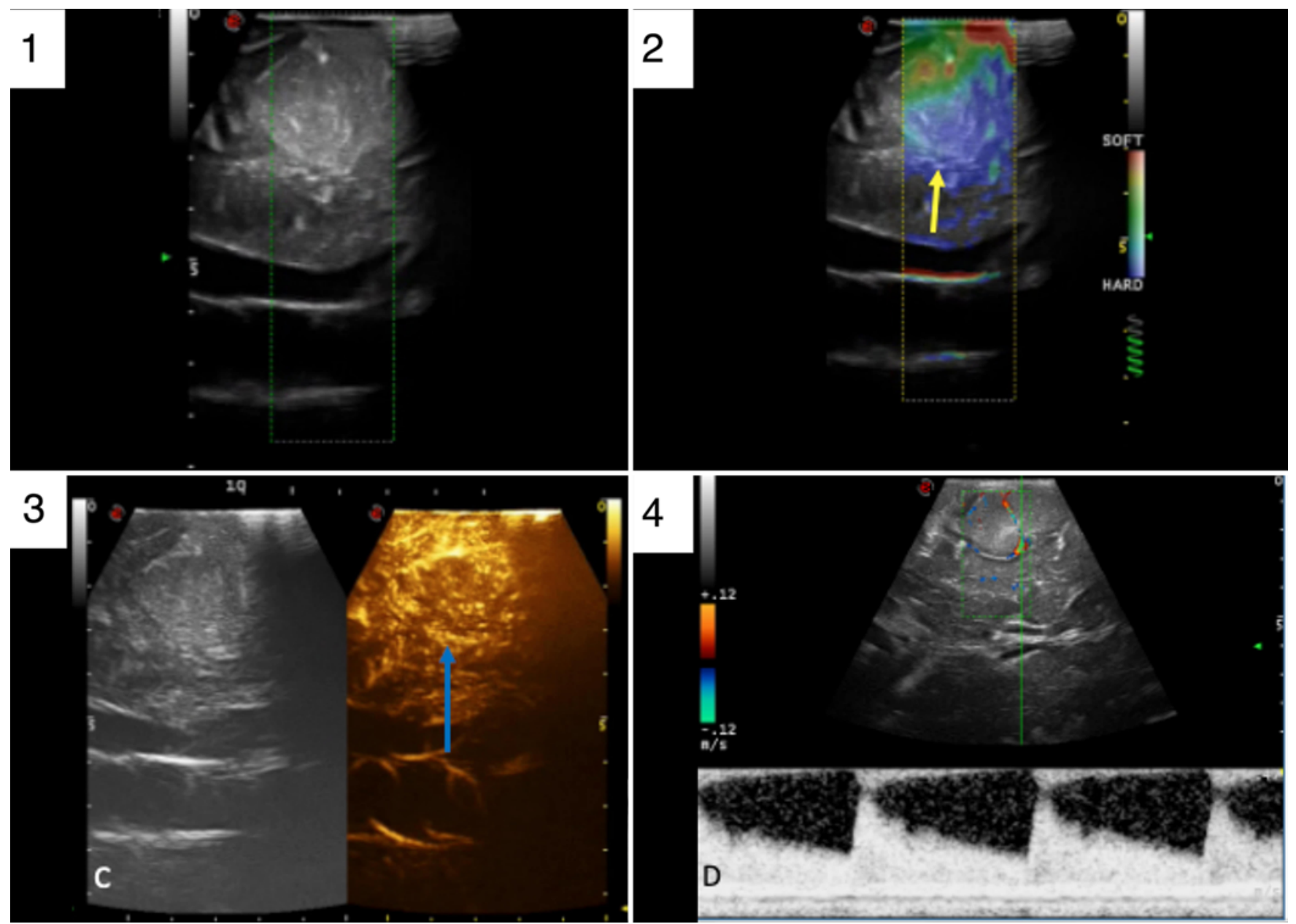

FIG. 2. A sequential integration of multiple US modalities can be effective in guiding surgical strategy, especially in meningioma. A case of right frontal meningioma is presented. Standard ioUS B-mode of a right frontal anaplastic grade III meningioma can be seen (1). When an elastosonographic image is acquired (2), it shows intratumoral heterogeneous consistency and the presence of an adherent interface (arrow). Therefore, the surgeon can use these data to decide where to start dissecting, if the lesion should be internally debulked, and which types of instruments are best to use during the resection. If contrast medium is added (pre- [left] and post- [right] contrast enhancement, panel 3) intralesional enhancement and neovascularization of the brain-tumor interface (arrow) can be seen, further improving the surgical strategy. Lastly, Doppler US (4) is of paramount importance in assessing the relationship of the tumor to physiological brain vascularization: the image demonstrates the presence of a sulcal artery within the brain-tumor interface, where the tumor is adherent to both the brain and the vessels.

Similarly, in meningioma surgery, the presence of a preserved arachnoid plane with a CSF film implies that CSF can be mobilized with pulsation, and that when a region moves independently from another, a red ("soft") color is displayed in the corresponding area, showing a "slip interface." While some experiences showed a good correlation between SUE and meningioma consistency, ${ }^{19}$ the technique has not yet been specifically investigated for meningiomas and its applicability in a surgical setting is unknown.

\section{Significance of ioUS Elastography in Guiding Surgical Strategy}

Elastographic assessment, similarly to standard Bmode US evaluation, presents several crucial advantages: 1) it is a quick tool, which avoids significant interruption of the surgical workflow; and 2) it is cost-effective, because it does not require expensive or specific equipment and the elastographic mode is already present on many US devices. Rapidity, cost-effectiveness, the possibility to study unexposed areas intraoperatively, and integrability with other techniques (Fig. 2) are only some of the advantages of ioUS elastography, which will be further discussed below in relation to meningioma consistency discrimination and assessment of meningioma-brain interface (Fig. 3).

\section{Consistency}

The intralesional SUE signal suggests the consistency of the tumor. Through a color map, the surgeon can test the consistency of the tumor in advance and adjust the surgical strategy accordingly. This would determine whether the lesion should be internally debulked and which types of instruments should be used for resection. Moreover, as shown in our series, different consistencies are often dis- 

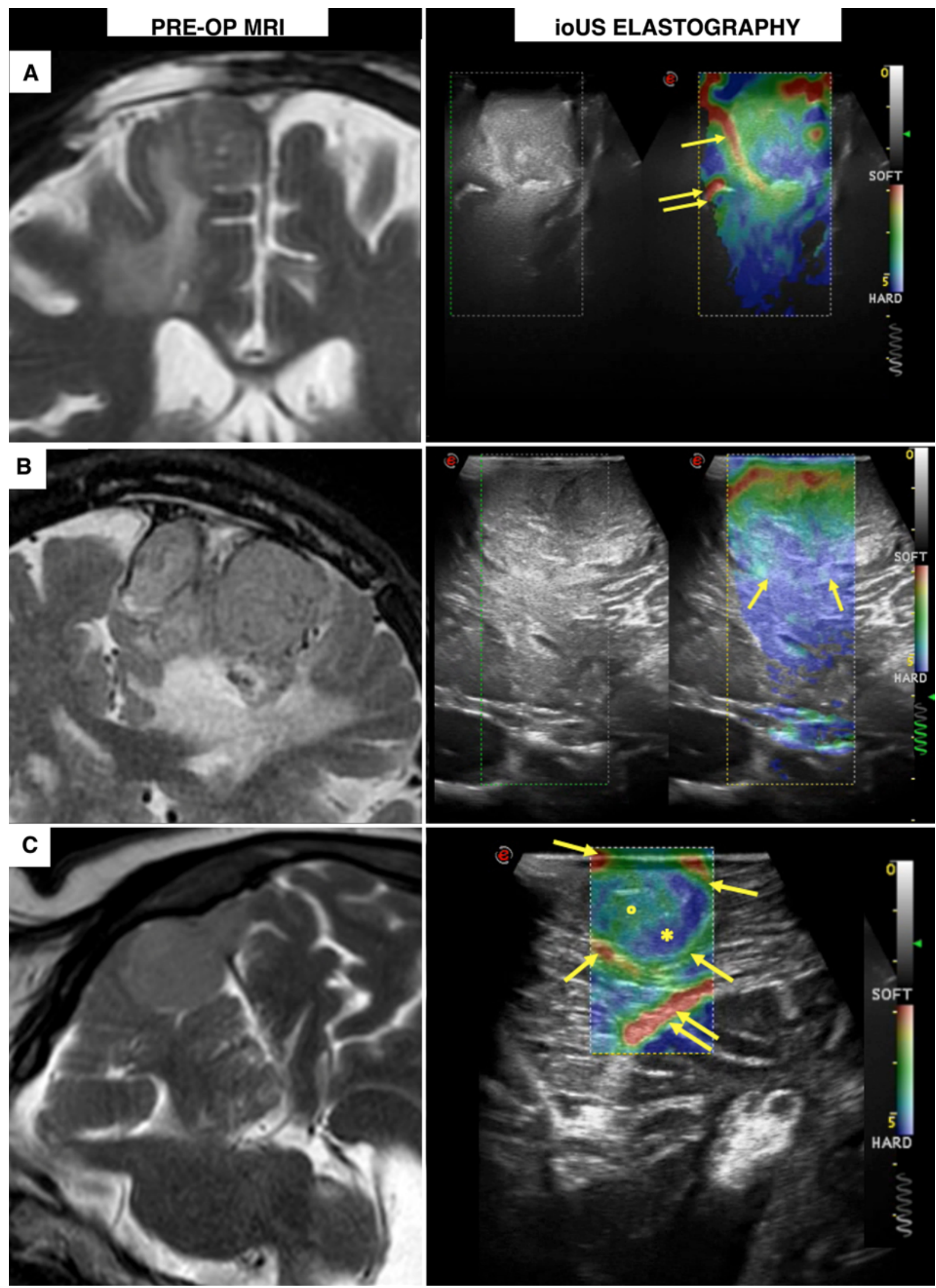

FIG. 3. Meningioma consistency and meningioma-brain interface are major determinants in defining the surgical strategy, especially if considered in relation to critical cortical areas and vascular structures. ioUS elastography results are particularly valuable for tumors that demonstrate extremes of consistency (i.e., extremely soft vs extremely firm) and adherent slip-brain interface. FIG. 3. (continued) $\rightarrow$ 
FIG. 3. Preoperative MRI features and elastographic and intraoperative findings are directly compared for three paradigmatic cases. In this series, what should be noticed is the different appearance of slip-brain interface at ioUS, whether it is preserved (case 1), adherent (case 2), or heterogenous (case 3). A: Case 1 (upper row) is a parietal meningothelial meningioma (WHO grade I, 2016), which does not present a proper interface at preoperative MRI (left). This finding, however, is proved incorrect during ioUS elastography and intraoperatively, after dural opening. An elastosonographic image (right) shows intratumoral heterogeneous consistency and the presence of a good slip interface (single arrow); as a proof of principle, the CSF within a subarachnoid space outside the tumor, where it is free to move and has a minimal consistency, shows a signal similar to the slip interface (double arrows). B: Case 2 involves an anaplastic meningioma (WHO grade III, 2016). An elastosonographic image (right) shows the presence of an adherent interface (arrows), confirming MRI findings (left). These results are displayed in the elastosonographic map as limited freedom of movement of the CSF in the arachnoid compartment at the interface, resulting in a "rigid," blue-colored area. In our series, a particularly adherent slip-brain interface at the elastographic map is particularly associated with infiltration of the arachnoid compartment, evident in grade II and III meningiomas. C: Case 3 (Iower row) presents as a meningioma with heterogenous consistency, from soft $\left({ }^{\circ}\right)$ to hard $\left({ }^{*}\right)$, and the presence of a mixed interface (single arrows) at ioUS elastography (right). As a demonstration of this principle, the CSF within a subarachnoid space outside the tumor, where it is free to move and has a minimal consistency, shows a signal similar to the slip interface (double arrows). This information is extremely helpful in planning the resection.

played within the same meningioma. This information can be used to develop a step-by-step approach, possibly debulking the softer areas before the harder ones.

\section{Interface}

In our experience, the interface is the feature for which the SUE map is the most relevant in guiding the surgical strategy. Three considerations have to be made. First, CSF within the brain-tumor interface has an expectedly very low density and it is very easy to displace when compression is applied, resulting in a very red color on the SUE map. Its presence is not homogeneous around the tumor, so that different types of brain-tumor interfaces are possible (e.g., mostly preserved, mostly mixed, mostly adherent). By assessing the type of brain-tumor interface using the SUE map, the surgeon can build his or her surgical strategy, i.e., starting tumor dissection from the better-preserved interface/most represented slip interface.

A second consideration is related to the degree of adherence of tumors in proximity to cortically eloquent areas. In such cases, the dissection can be performed only after debulking: knowing the edges with more favorable margins could be of paramount importance in deciding where to start the dissection. A third consideration is related to vascular preservation: when it comes to dissecting the pathology from surrounding vessels, especially if these are deeply located or unexposed, precise knowledge of the degree of adherence in different areas of the meningioma might be crucial in defining the overall strategy.

\section{ioUS Elastography: Intraoperative Use}

Preoperative MRI is of the utmost importance in enabling accurate preoperative surgical planning. Specifically, it provides a precise knowledge of the anatomical location of the brain lesion (determining patient positioning) and a deep comprehension of its spatial relationship to the surrounding healthy tissue. Assuming that the surgical strategy is entirely based on MRI data, it should be noted that poor agreement was reported between preoperative MRI predictions and surgical findings with regard to meningioma consistency and interface (ICC $=0.006, \mathrm{p}$ $=0.486$, and ICC $=0.418, p=0.006$, respectively). Because timely information on the abovementioned characteristics could help the surgeon to redesign the strategy intraoperatively (after craniotomy but before starting dissection), in this context ioUS elastography could represent a useful complementary tool. Despite the fact that ioUS elastography is not conclusive by itself, our experience demonstrated that it can provide useful additional data when added to the routine intraoperative ecographic assessment and facilitate changes in surgical strategy.

Surgeons must choose which side to begin debulking from; starting from the softer side reduces the pressure applied to the meningioma and, consequently, to the brain. In our experience, ioUS elastography has proved to be accurate (more so than preoperative MRI) in identifying softer areas, and without the need to directly test consistency (ICC $=0.848, p<0.001)$. In our series, some meningiomas for which a piecemeal resection was planned due to an expected soft consistency, showed a different consistency at elastography. In those cases, the surgical strategy was changed accordingly (switching from piecemeal to en bloc resection).

Similarly, surgeons prefer to start dissecting from a well-preserved interface. This information is generally gathered from MRI, where the presence of a surrounding CSF film is shown as a hyperintense signal on T2-weighted imaging. However, we demonstrated that MRI is not sufficiently reliable in predicting brain-meningioma interface, which is more effectively assessed by ioUS elastography (ICC $=0.418, p=0.006)$. When elastography is feasible, the surgeon is able to adjust his or her strategy in real-time and optimize tumor dissection beginning from the actual better-preserved interface.

The aforementioned examples are summarized and detailed in Fig. 4. To summarize, ioUS elastography should not be regarded as an instrument to be used on its own. Our surgical experience suggests its greatest advantage is its fast and easy integration with both preoperative data (MRI) and other intraoperative ecographic assessment tools. Therefore, the role of elastography is to be an additional tool in the routine ecographic assessment that is already performed intraoperatively in many centers, as it can be quickly integrated with other US-driven data such as color Doppler, power Doppler, or contrast-enhanced US, providing surgeons with some additional information before starting the central phase of surgery that can be valuable (as mentioned) in lesions close to eloquent areas, sulcal vessels, dural sinuses, or deep veins. ${ }^{20,21}$ It is important to underline that the technique is ancillary and has to be considered as an extra tool in intraoperative imaging assessment of these lesions. 


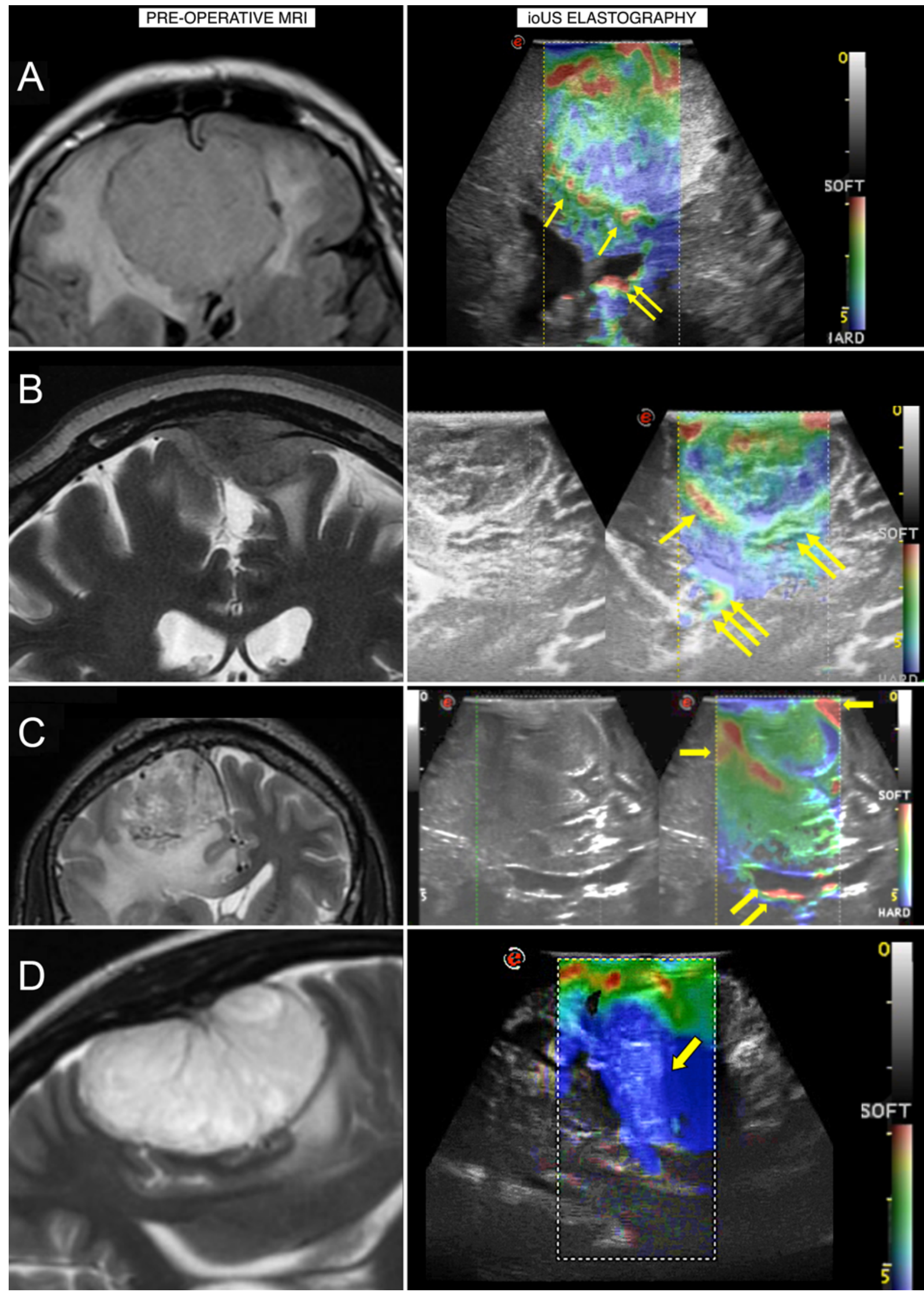

FIG. 4. The SUE modality of US elastography has the advantage of being a real-time examination, allowing the step-by-step adjustment of surgical strategy once consistency and interface information are gathered. FIG. 4. (continued) $\rightarrow$ 
FIG. 4. This is significant, especially whether the consistency or interface is mixed, as demonstrated by cases $A$ and $B$ in the figure. A: Case A is an ethmoidal meningioma as seen at preoperative MRI (left) and ioUS elastography (right). An elastosonographic image shows an intratumoral heterogenous consistency, which is significant from the surgical point of view because it is advisable to start debulking from the softer areas instead of harder ones, and the presence of a good interface (single arrows). As a proof of principle, the CSF within a subarachnoid space outside the tumor, where it is free to move and has a minimal consistency, shows a signal similar to the slip interface (double arrows). B: Case B depicts a parietal meningioma. It is relevant because the elastosonographic image shows a mixed interface, good (single arrow), or adherent (double arrows). By assessing the type of brain-tumor interface using the SUE map, the surgeon can build his or her surgical strategy, i.e., starting the tumor dissection from the better-preserved interface to the most adherent one. Moreover, if a mismatch between MRI and elastographic findings is found, a successful change in surgical strategy can be performed in a timely manner, as demonstrated by cases $\mathrm{C}$ and D. C: Case $\mathrm{C}$ is a parasagittal meningioma. While at preoperative MRI the meningiomabrain interface appeared adherent (left), ioUS elastography (right) demonstrated it as good (single arrows). As a proof of principle, the CSF within a subarachnoid space outside the tumor shows a signal similar to the slip interface (double arrows). This information can help the surgeon to intraoperatively replan the surgical strategy, rapidly identifying the actual well-preserved interface to begin the debulking. D: Case $D$ is a convexity meningioma. It is relevant because, different from the MRI (left), elastography (right) demonstrated an overall hard tumor texture (single arrow). If this information was not available, the surgeon would not have changed the surgical strategy (from the planned piecemeal resection to an en bloc resection), without the need to directly test consistency.

\section{Reliability of the ioUS Elastographic Map}

The experience of 36 cases presented here is currently the largest series in the literature investigating ioUS elastography in meningiomas, and the first study to specifically focus on brain-meningioma interface. ioUS elastography was effectively applied, allowing the acquisition of real-time high-quality data about the explored anatomy. To demonstrate this, a good correlation was found between elastographic and surgical scores, either in the case of meningioma-brain interface or overall meningioma texture. In particular, when analyzing both consistency and brain-tumor interface, ioUS elastography proved to have a rather elevated sensitivity, specificity, PPV, NPV, LR+, and LR-, and appeared to be more reliable than MRI. This consideration remains true especially for hard-consistency meningiomas and those presenting with an adherent slipbrain interface.

Interestingly, although a strict correlation cannot be demonstrated, a trend between elastographic patterns of interface and meningioma histopathology was identified. Infiltration of the arachnoid compartment and pial layers was more evident in WHO grade II and III meningiomas, resulting in limited freedom of movement at the interface. In our series, this corresponded to a particularly adherent slip-brain interface at the elastographic map.

\section{Implications and Limitations}

US elastography is an intraoperative technique that allows for the identification of morpho-elastic characteristics of intracranial structures before the dura is opened and makes their assessment possible before structures are surgically exposed. As previously stated, the SUE modality of US elastography has the undeniable advantage of being a prompt and real-time examination, without the need for any postprocessing elaboration (Fig. 4). However, this is different from true presurgical planning, as it is performed intraoperatively only after the bone flap is removed.

Moreover, this tool is limited by the nonquantitative data that it can provide, different from other modalities such as shear-wave elastography ${ }^{22}$ that, in contrast, requires postprocessing of the acquired images. The aforementioned potential issue was also reported by Prada et al., who stated that, in common practice, data obtained with nonquantitative methods can be as useful as those obtained by quantitative methods when it comes to glioma management. ${ }^{15}$ The technique, because of its operatordependent nature, may appear complex to nonexpert neurosurgeons; this is related to the difficulties in obtaining images compatible with the standard radiological planes and in keeping correct intraoperative spatial orientation as well. Further limitations to our study can be noted, i.e., it is a prospective series of a relatively small number of patients and it includes mostly cranial vault meningiomas.

\section{Conclusions}

The series presented here is the largest in the literature investigating the role of ioUS elastography in meningioma surgery. Our observations first demonstrated that elastosonographic characteristics are predictive of key intracranial meningiomas features, namely meningioma consistency and brain-meningioma interface assessment. This consideration remains true especially for hard-consistency meningiomas and those presenting with an adherent slipbrain interface. Second, it was proved that these meningiomas features, assessed after craniotomy but before dural opening, can guide surgical strategy. Lastly, it showed that ioUS has a higher agreement with surgical findings when compared to other available techniques. While external validation of the data is needed to turn it into a fully clinical deployable tool, our experience confirmed that ioUS elastography may be used in meningioma surgical planning by virtue of its rapidity and cost-effectiveness. Finally, elastographic maps in meningiomas can, hence, be considered as an additional corollary tool to the standard ioUS assessment of meningiomas.

\section{References}

1. Shiroishi MS, Cen SY, Tamrazi B, et al. Predicting meningioma consistency on preoperative neuroimaging studies. Neurosurg Clin N Am. 2016;27(2):145-154.

2. Yin Z, Hughes JD, Trzasko JD, et al. Slip interface imaging based on MR-elastography preoperatively predicts meningioma-brain adhesion. J Magn Reson Imaging. 2017;46(4): 1007-1016.

3. Chartrain AG, Kurt M, Yao A, et al. Utility of preoperative meningioma consistency measurement with magnetic resonance elastography (MRE): a review. Neurosurg Rev. 2019; 42(1):1-7.

4. Cepeda S, Barrena C, Arrese I, et al. Intraoperative ultrasonographic elastography: a semi-quantitative analysis of brain tumor elasticity patterns and peritumoral region. World Neurosurg. 2020;135:e258-e270. 
5. Scholz M, Noack V, Pechlivanis I, et al. Vibrography during tumor neurosurgery. J Ultrasound Med. 2005:985-992.

6. Dietrich CF, Barr RG, Farrokh A, et al. Strain elastographyHow to do it? Ultrasound Int Open. 2017;3(4):E137-E149.

7. Murphy MC, Huston J III, Ehman RL. MR elastography of the brain and its application in neurological diseases. Neuroimage. 2019;187:176-183.

8. Yin Z, Romano AJ, Manduca A, et al. Stiffness and beyond: what MR elastography can tell us about brain structure and function under physiologic and pathologic conditions. Top Magn Reson Imaging. 2018;27(5):305-318.

9. Wildeboer RR, Mannaerts CK, van Sloun RJG, et al. Automated multiparametric localization of prostate cancer based on B-mode, shear-wave elastography, and contrast-enhanced ultrasound radiomics. Eur Radiol. 2020;30(2):806-815.

10. Zhao C-K, Xu H-X. Ultrasound elastography of the thyroid: principles and current status. Ultrasonography. 2019;38(2): $106-124$.

11. Liu H, Wan J, Xu G, et al. Conventional US and 2-D shear wave elastography of virtual touch tissue imaging quantification: correlation with immunohistochemical subtypes of breast cancer. Ultrasound Med Biol. 2019;45(10):2612-2622.

12. Lu C, Merrill C, Medellin A, et al. Bowel ultrasound state of the art: grayscale and Doppler ultrasound, contrast enhancement, and elastography in Crohn disease. J Ultrasound Med. 2019;38(2):271-288

13. Kawada N, Tanaka S. Elastography for the pancreas: current status and future perspective. World J Gastroenterol. 2016; 22(14):3712-3724.

14. Chauvet D, Imbault M, Capelle L, et al. In vivo measurement of brain tumor elasticity using intraoperative shear wave elastography. Ultraschall Med. 2016;37(6):584-590.

15. Prada F, Del Bene M, Rampini A, et al. Intraoperative strain elastosonography in brain tumor surgery. Oper Neurosurg (Hagerstown). 2019;17(2):227-236.

16. Selbekk T, Solheim O, Unsgård G. Ultrasound-guided neurosurgery: experiences from 20 years of cross-disciplinary research in Trondheim, Norway. Neurosurg Focus. 2016;40(3): E2.

17. Selbekk T, Brekken R, Solheim O, et al. Tissue motion and strain in the human brain assessed by intraoperative ultrasound in glioma patients. Ultrasound Med Biol. 2010;36(1): $2-10$.
18. Uff CE, Garcia L, Fromageau J, et al. Real-time ultrasound elastography in neurosurgery. Presented at the 2009 IEEE International Ultrasonics Symposium; September 20-23, 2009; Rome, Italy.

19. Prada F, Del Bene M, Moiraghi A, et al. From grey scale Bmode to elastosonography: multimodal ultrasound imaging in meningioma surgery-pictorial essay and literature review. BioMed Res Int. 2015;2015:925729.

20. Della Pepa GM, Menna G, Ius T, et al. Contrast enhanced ultrasound (CEUS) applications in neurosurgical and neurological settings-New scenarios for brain and spinal cord ultrasonography. A systematic review. Clin Neurol Neurosurg. 2020;198:106105.

21. Della Pepa GM, Ius T, La Rocca G, et al. 5-Aminolevulinic acid and contrast-enhanced ultrasound: the combination of the two techniques to optimize the extent of resection in glioblastoma surgery. Neurosurgery. 2020;86(6):E529-E540.

22. Chan HW. Optimising the Use and Assessing the Value of Intraoperative Shear Wave Elastography in Neurosurgery [doctoral thesis]. London: University College London; 2016.

\section{Disclosures}

The authors report no conflict of interest concerning the materials or methods used in this study or the findings specified in this paper.

\section{Author Contributions}

Conception and design: Della Pepa. Acquisition of data: Della Pepa, Stifano. Analysis and interpretation of data: Della Pepa, Menna. Drafting the article: Della Pepa, Menna, Stifano.

Critically revising the article: Menna, Stifano, Auricchio, Caccavella, La Rocca, Sabatino. Reviewed submitted version of manuscript: Menna, Stifano, Marchese, Olivi. Statistical analysis: Pezzullo. Administrative/technical/material support: Rapisarda. Study supervision: Della Pepa, Olivi.

\section{Correspondence}

Giuseppe Maria Della Pepa: Fondazione Policlinico Universitario A. Gemelli IRCCS, Università Cattolica del Sacro Cuore, Rome, Italy.gdellapepa@hotmail.com. 